\title{
Pé Diabético: onde podemos intervir?
}

Diego De Matos Silveira*

Lize Vargas Ferreira**

Guilherme Henrique Werner Sathler Fraga*

Isabela Silva de Sousa*

Mônica Barros Costa**

\section{RESUMO}

Trata-se de estudo descritivo, em coorte transversal incluindo 59 indivíduos com DM, que apresentavam pé diabético como complicação, atendidos no ambulatório de Pé Diabético do Centro Hiperdia, no município de Juiz de Fora - MG, no período de julho de 2013 a julho de 2014. Resultados: As condições predisponentes mais prevalentes foram HAS $(96,6 \%)$, neuropatia $(85,4 \%)$ e dislipidemia $(69,5 \%)$. Foi observada ainda presença de sobrepeso/obesidade $(28,8 \% / 50,8 \%)$, tabagismo $(42,4 \%)$, sedentarismo $(28,8 \%)$ e mau controle glicêmico (HbA1c: 8,8\% $\pm 2,24$ ). Em relação ao cuidado com os pés, 86,4\% dos participantes se diziam conscientes. É fundamental a instituição da educação em diabetes em todos os níveis de cuidado, para que a prevenção se dê de forma realmente eficaz, visto que a maioria dos fatores de risco são modificáveis. A orientação desses pacientes sobre cuidado com os pés é um importante instrumento na redução do risco de ulceração e amputação em membros inferiores.

Palavras-chaves: Diabetes mellitus. Pé diabético. Fatores de risco. Autocuidado.

\section{INTRODUÇ̃̃o}

Diabetes mellitus (DM) pode ser definido como uma síndrome constituída por distúrbios metabólicos que apresentam em comum a hiperglicemia, resultado de defeitos na ação ou na secreção de insulina ou em ambas (OLIVEIRA;VENCIO, 2013/2014). Essa síndrome vem alcançando altos índices de morbimortalidade no mundo, sendo importante causa de óbito por condição crônica de saúde, seja como causa básica ou associada (PACE et al., 2002).

ODM é uma enfermidade que pode ser controlada, porém são necessárias mudanças nos hábitos de vida. Os diabéticos devem adotar certos comportamentos específicos de auto cuidado, e também receber ações de vigilância e assistência à saúde para que a glicemia seja mantida o mais próximo possível do nível apropriado (SANTOS et al., 2005), (GROSS; NEHME, 1999).

Se não controlado, o diabetes pode acarretar complicações agudas, tais como hipoglicemia, cetoacidose diabética e coma hiperosmolar, e crônicas, como transtornos na microcirculação, causando retinopatia, neuropatia e nefropatia e na macrocirculação, levando a cardiopatia isquêmica, doença vascular periférica e doença cérebro-vascular.

Dentre as complicações crônicas é de grande relevância o pé diabético, que é definido como infecção, ulceração e/ou destruição dos tecidos profundos associadas a anormalidades neurológicas e vários graus de doença vascular periférica nos membros inferiores (SANTOS et al., 2005). Dependendo da evolução clínica desses sintomas e do grau de comprometimento, a amputação do membro inferior pode ser necessária. O pé diabético é uma das mais importantes e devastadoras complicações do diabetes mellitus, sendo a causa mais comum de amputações não traumáticas em membros inferiores (OLIVEIRA;VENCIO, 2013/2014), (PACE et al., 2002).

No Brasil, 484.500 úlceras são estimadas em um modelo hipotético de uma população de 7,12 milhões de pessoas com diabetes mellitus do tipo 2 (DM2), com 169.600 admissões hospitalares e 80.900 amputações efetuadas, das quais 21.700 evoluíram para morte. Os custos anuais hospitalares são estimados em 461 milhões de dólares (OLIVEIRA; VENCIO, 2013/2014).

O presente trabalho tem por objetivo avaliar características clínicas e laboratoriais, controle metabólico, cuidado com os pés e presença de outras complicações em portadores do pé diabético.

\footnotetext{
* Universidade Federal de Juiz de Fora-MG

** Serviço de endocrinologia do Hospital Universitário da Universidade Federal de Juiz de Fora
} 


\section{Material e Métodos}

Foi realizado estudo descritivo, em coorte transversal incluindo 59 indivíduos com DM, que apresentavam pé diabético como complicação, atendidos no ambulatório de Pé Diabético do Centro Hiperdia, no município de Juiz de Fora - MG, no período de julho de 2013 a julho de 2014.

Os participantes da pesquisa foram abordados pelos pesquisadores na própria instituição, quando receberam informações detalhadas sobre o estudo, através de exposição dialogada e foram convidados a participar do mesmo. Os que concordaram em participar e tiveram o Termo de Consentimento Livre e Esclarecido assinado foram abordados individualmente e entrevistados em local reservado na própria instituição.

A avaliação constou da pesquisa de dados pessoais do paciente (nome, data de nascimento, sexo, escolaridade e renda familiar aproximada), dados clínicos coletados dos prontuários dos pacientes (história do diabetes, presença de hipertensão arterial sistêmica, perfil metabólico, e índice de massa corpórea - IMC mais recentes). Foi pesquisado sedentarismo segundo Questionário Internacional de Atividade Física versão curta (IPAQ) (CESCHINI et al., 2009), tabagismo segundo questionário de tolerância de Fagerström (MENEZES et al., 2009), grau de dependência alcóolica segundo CAGE (FILHO et al., 2011) e cuidado com os pés (MICHELS et al., 2010).

Além disso, foi rastreada a presença de fatores de risco para complicações em membros inferiores, dentre eles úlcera prévia, neuropatia periférica, calosidade, deformidade, ausência de pulsos tibial posterior/pedioso, micose, bolhas, rachaduras, fissuras (GROSS; NEHME, 1999), amputação classificada em menor e maior (BRASILEIRO et al., 2005), (NUNES et al., 2006), através de exame físico e coleta de dados de prontuário da Enfermagem, Neurologia, Endocrinologia e Angiologia. Os pacientes também foram avaliados quanto ao índice tornozelo-braço (ITB) (CUNHA et al., 2007), (MAKDISSE et al., 2007) e ao uso de calçados inapropriados no momento da entrevista (PACE et al., 2002). Foram considerados calçados inapropriados quando os mesmos eram apertados, de bico fino, abertos e sem cadarço. Entre as mulheres, além das características apontadas, foram consideradas inapropriadas as sandálias que deixam totalmente expostos os pés, os saltos maiores de três centímetros e aqueles extremamente largos e/ou compridos.

Tais resultados foram tratados com recursos da Estatística básica (formulação de quadros e tabelas, desenho de gráficos, identificação de medidas de distribuição de freqüência, percentuais, média, com seus desvios, conforme os casos).

O estudo foi aprovado pelo Comitê de Ética em Pesquisa CEP/UFJF, de acordo com as atribuições definidas na Res. CNS 196/96, mediante parecer $\mathrm{n}^{\circ}$ 172.998, na data 13/12/2012.

\section{Resultados}

No estudo foram avaliados 59 pacientes, sendo 32 mulheres (54,2\%) e 27 homens (46,8\%), entre 38 e 86 anos de idade (média de 62,5 $\pm 11,20$ ). Esses pacientes tinham DM diagnosticado há $15 \pm 9,4$ anos.

No gráfico 1 estão descritas as características identificadas na população do estudo referente ao grau de escolaridade.

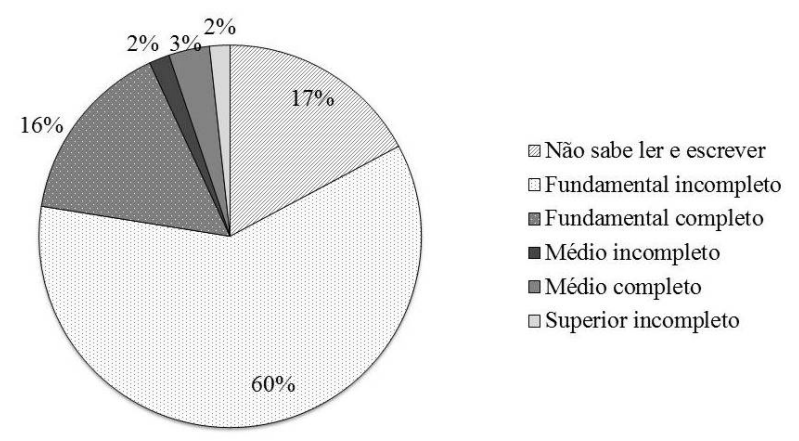

Gráfico 1: Grau de escolaridade.

Fonte: Os autores (2012).

Em relação à renda familiar, foi constatado que $31 \%$ dos participantes apresentavam renda de 1 salário mínimo, $42 \%$ de 1 a 2 salários mínimos e $27 \%$ de 2 a 5 salários mínimos.

$\mathrm{Na}$ Tabela 1 estão descritas as características comportamentais encontradas nos indivíduos do estudo.

\section{TABELA 1}

Características comportamentais dos indivíduos estudados $(n=59)$

\begin{tabular}{lcc}
\hline Características & N & Percentual \\
\hline Tabagismo & 7 & 11,9 \\
Ex-tabagismo & 18 & 30,5 \\
Sedentarismo & 17 & 28,8 \\
Uso de calçado inapropriado & 20 & 33,9 \\
\hline
\end{tabular}

Fonte: Os autores (2012).

Nos tabagistas foi encontrada carga tabágica média de 24,8 anos/maço e nos ex-tabagistas, 33,1 anos/maço.

$\mathrm{Na}$ Tabela 2 estão descritas as principais comorbidades relacionadas a maior risco de ulceração em membros inferiores de portadores de DM. 
TABELA 2

Principais comorbidades encontradas na população estudada

$$
(n=59)
$$

\begin{tabular}{lcc}
\hline Características & N & Percentual \\
\hline Sobrepeso/Obesidade & $17 / 30$ & $28,8 / 50,8$ \\
HAS & 57 & 96,6 \\
Dislipidemia & 41 & 69,5 \\
$\begin{array}{l}\text { Doença vascular periférica } \\
(\text { ITB* alterado }(<0,9)\end{array}$ & 27 & 45,7 \\
\hline
\end{tabular}

*ITB - Índice Tornozelo-Braço.

Fonte: Os autores (2012).

Os dados de sobrepeso e obesidade foram obtidos através do cálculo de IMC, sendo encontrada média de $30,7 \pm 6,72 \mathrm{~kg} / \mathrm{m}^{2}$.

$\mathrm{Na}$ Tabela 3 se encontram os valores mais recentes relacionados ao perfil metabólico.

\section{TABELA 3}

Características laboratoriais dos indivíduos estudados $(n=59)$

\begin{tabular}{lc}
\hline Características & Média \pm Desvio padrão \\
\hline Glicose em jejum (mg/dL) & $160 \pm 75,6$ \\
Hemoglobina glicada (\%) & $8,8 \pm 2,24$ \\
Colesterol total (mg/dL) & $196 \pm 57,1$ \\
Colesterol LDL (mg/dL) & $110 \pm 42,9$ \\
Colesterol HDL (mg/dL) & $46 \pm 10,6$ \\
Triglicérides (mg/dL) & $197 \pm 147,6$ \\
\hline Fonte: Os autores (2012). &
\end{tabular}

Fonte: Os autores (2012).

Foram identificadas, através do exame físico, alterações que representam fatores de risco para complicações nos pés dos participantes do estudo, e

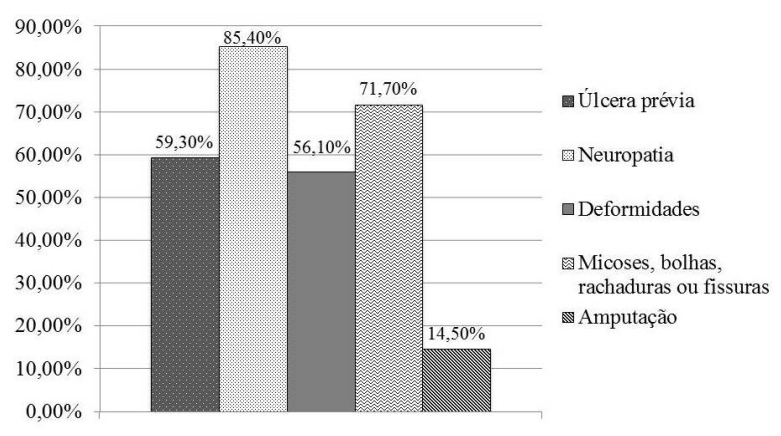

Gráfico 3: Características encontradas no exame físico. Fonte: Os autores (2012).

estão descritas no Gráfico 3.

Em relação ao cuidado com os pés, 86,4\% (n=51) dos participantes se diziam conscientes, examinandoos todos os dias, $83 \%(\mathrm{n}=49)$ disseram que examinaram dentro dos sapatos antes de calçá-lo todos os dias, e $100 \%(\mathrm{n}=59)$ dos entrevistados disseram secar os espaços entre os dedos dos pés depois de lavá-los todos os dias, na semana anterior a entrevista.

\section{DiscuSSÃo}

$\mathrm{Na}$ amostra do estudo, observou-se que $73 \%$ da população estudada possuem renda familiar de até dois salários mínimos, compatível com o perfil dos usuários do SUS. Além disso, 17\% não sabem ler ou escrever e $60 \%$ não concluiu o ensino fundamental. A baixa escolaridade pode dificultar o processo de busca e acesso a informações, a compreensão dos mecanismos da doença e seu tratamento e o aprendizado em relação ao cuidado com a própria saúde (ROCHA et al., 2009).

As características comportamentais abordadas no estudo foram tabagismo, sedentarismo e uso de calçado inapropriado. No presente estudo, extabagistas e tabagistas totalizaram $42,4 \%$ da amostra, corroborando com os dados encontrados em estudo realidado em 2009 em Ambulatório de Hospital Universitário e em duas Unidades Básicas de Saúde de pequenos municípios situados na região norte do estado do Paraná, no qual 42,5\% dos pacientes eram tabagistas (BORTOLETTO; HADDAD; KARINO, 2009). O tabagismo está relacionado principalmente com a formação de úlceras em membros inferiores, uma vez que é considerado um fator agravante da doença aterosclerótica vascular periférica em diabéticos (BORTOLETTO; HADDAD; KARINO, 2009).

O sedentarismo foi avaliado segundo o Questionário Internacional de Atividade Física versão curta (IPAQ) (CESCHINI et al., 2009). Nesse estudo, a maioria dos pacientes $(71,2 \%)$ foi considerada ativa fisicamente e $28,8 \%$ dos pacientes foram classificados como sedentários. Tal fato pode-se relacionar ao atendimento de referência oferecido aos pacientes estudados através dos ambulatórios de hipertensão arterial, nefropatia diabética, obesidade, síndrome metabólica, cuidados com o pé diabético, os quais incentivam a mudança de estilo de vida a fim de alcançar o objetivo da terapêutica. Um estudo realizado em um centro de pesquisa e extensão universitária do interior paulista em 2005, acompanhado de projetos educativos, $65 \%$ dos pacientes praticavam algum tipo de atividade física, salientando a importância das intervenções educativas em saúde (ROCHA et al., 2009).

As principais comorbidades associadas a risco de ulceração são sobrepeso/obesidade, dislipidemia, hipertensão, dislipidemia e vasculopatia periférica, avaliada pelo índice tornozelo-braço (ITB). A obesidade é uma epidemia mundial e o Brasil tem cerca de 18 milhões de pessoas consideradas obesas. É fator de risco para hipertensão arterial sistêmica, doença cardiovascular e DM2. No presente estudo, o sobrepeso/obesidade foi a terceira comorbidade mais

HU Revista, Juiz de Fora, v. 43, n. 1, p. 13-18, jan./jun. 2017 
prevalente $(79,7 \%)$, sendo que $28,8 \%$ dos pacientes apresentaram sobrepeso e 50,8\% apresentaram obesidade, comparando a outros estudos, como o realizado no interior paulista em 2009 onde 63,6\% da população se encontrava obesa, destacando a alta prevalência dessa comorbidade (ROCHA et al., 2009)

A hipertensão arterial sistêmica (HAS) esteve presente em 96,6\% dos pacientes da amostra, dado que pode ser explicado pelo fato de o estudo ter sido realizado em um centro especializado em Diabetes e Hipertensão. A HAS é uma comorbidade duas vezes mais frequente nos indivíduos diabéticos, comparados à população geral, sendo que esta frequência aumenta com a idade. Estudos e consensos brasileiros mostram que a associação de HAS e DM é da ordem de $50 \%$, o que leva ao paciente diabético um fator de risco adjuvante, já que altos níveis pressóricos contribuem para o aparecimento das complicações macro e microvasculares. Por serem pacientes de alto risco para eventos cardiovasculares, recomenda-se que todos os indivíduos diabéticos mantenham níveis pressóricos controlados abaixo ou igual a 130x80 mmHg. (BORTOLETTO; HADDAD; KARINO, 2009), (OLIVEIRA;VENCIO, 2013/2014).

O índice tornozelo-braço é uma avaliação quantitativa, simples e não invasiva capaz de identificar e avaliar a gravidade da doença arterial obstrutiva periférica. No estudo $45,7 \%$ dos pacientes possuiam ITB alterado (menor que 0,9 ) apresentando risco de incapacidade funcional e amputação dos membros acometidos (NORGREN et al., 2007).

Dos pacientes estudados, 69,5\% apresentam dislipidemia. Essa alta prevalência se compara a outros estudos, como o realizado em um centro de pesquisa e extensão universitária do interior paulista, em 2005, onde $49 \%$ dos participantes apresentavam dislipidemia.(15) A dislipidemia é provavelmente o principal fator de risco para o desenvolvimento de doenças aterotrombóticas, contribuindo para a doença arterial periférica e representando maior risco para ulceração dos membros inferiores. Além disso, a dislipidemia tem efeitos tóxicos nas células $\beta$ pancreáticas e, associada à hiperglicemia, aumenta potencialmente o risco cardiovascular (OLIVEIRA;VENCIO, 2013/2014).

Em relação ao controle metabólico, constatou-se controle glicêmico inadequado (média de glicemia de jejum $160 \mathrm{mg} / \mathrm{dL}$ e HbA1c 8,8\%) e dislipidemia com colesterol HDL baixo $(46 \mathrm{mg} / \mathrm{dL})$ e triglicerídeos altos $(197 \mathrm{mg} / \mathrm{dL})$, típica do paciente com DM 2. Tais dados se aproximam aos encontrados em um estudo realizado no Ambulatório de Diabetes de um Hospital Universitário em Ribeirão Preto - SP, no qual a média de glicemia de jejum foi de $143 \mathrm{mg} / \mathrm{dL}, \mathrm{HbA1c}$
9,4\% e HDL $42 \mathrm{mg} / \mathrm{dL}$ ( MARTIN et al., 2012). O tempo médio de diagnóstico do DM foi de 15 anos. Sabe-se que o longo tempo de duração da doença, associado ao mau controle, favorece a instalação e o desenvolvimento de complicações crônicas, dentre elas o pé diabético.

Estudos demonstram que a redução dos níveis de hemoglobina glicada (HbA1c) em adultos sem complicações significativas para valores $<7 \%$ pode diminuir as complicações microvasculares e, possivelmente, as complicações macrovasculares do diabetes. Além disso, de acordo com outros estudos, há relação direta entre os níveis de hemoglobina glicada e severidade de neuropatia diabética. Hiperglicemia e dislipidemia associadas podem contribuir também para o desenvolvimento de doença arterial periférica, importante fator de risco para o pé diabético, evidenciada principalmente por sintomas de claudicação intermitente e pelo índice tornozelobraço alterado (MARTIN et al., 2012), (CUBAS et al., 2013).

A neuropatia periférica, principal agente que inicia o processo fisiopatológico das alterações em membros inferiores de diabéticos, esteve presente em $85,4 \%$ dos pacientes neste estudo, sendo a segunda comorbidade mais frequente. Em um estudo realizado entre 1998 e 2002 no Hospital Universitário do Mato Grosso do Sul, a neuropatia foi considerada a etiologia mais prevalente no pé diabético. Tal comorbidade pode causar alterações tróficas na pele e estrutura osteoarticular do pé e atrofia muscular, que podem gerar deformidades (observadas em 56,1\% dos pacientes), diminuição na amplitude de movimento, culminando no aumento de pressões plantares. Além disso, o fato mais importante em relação a essa comorbidade é a perda da sensibilidade, que somada às alterações previamente citadas deixa o paciente mais vulnerável a traumas triviais nos pés. Esses traumas tornam-se porta de entrada para infecções que podem se agravar se não tratadas precocemente (BRASILEIRO et al., 2005).

Foram encontradas ainda, no exame físico, micose interdigital, bolhas, rachaduras ou fissuras em 71,7\%. Essas condições, associadas à má higiene, podem contribuir para o surgimento de lesões em membros inferiores, e interferir no processo de recuperação das lesões já existentes. O paciente que apresentou úlcera prévia $(59,3 \%)$ ou amputações $(14,5 \%)$ é considerado de risco muito alto de nova ulceração, reforçando a importância do acompanhamento desse paciente até observar melhora clínica dessas lesões. Apesar de alta frequência de úlceras prévias, o número de amputações é menor se comparado a outros estudos, como o realizado em Ribeirão Preto - SP que encontrou 
$33.3 \%$ de amputações e história de ulcerações em 53.3\% dos pacientes (MARTIN et al., 2012).

A prevenção é a principal ferramenta contra as úlceras diabéticas. Estudos demonstram que medidas educativas abrangentes, como exame regular dos pés e educação terapêutica podem diminuir a ocorrência de lesões nos pés em até 50\% (CUBAS et al., 2013). No presente estudo, os pacientes se mostraram cientes da importância do autocuidado em relação aos pés. $86,4 \%$ relataram examiná-los diariamente, $83 \%$ disseram que examinaram dentro dos sapatos antes de calçálo todos os dias, e 100\% dos entrevistados disseram secar os espaços entre os dedos dos pés depois de lavá-los todos os dias na semana anterior a entrevista. Entretanto, mesmo pacientes aderentes a medidas de auto-cuidado ainda apresentam lesões relacionadas ao pé diabético, o que reforça a necessidade de início precoce das medidas educativas.

Outro aspecto importante é que o uso de calçado inapropriado, associado ao autocuidado ineficaz, é uma das principais causas de ulceração, devido à pressão que o mesmo exerce sobre o pé, principalmente se este apresenta algum tipo de deformidade. Estudos apontam que os pontos de alta pressão, calosidades, deformidades nos pés, amputação de dedos, ou mesmo transmetatarsianas, são problemas que podem ser corrigidos com calçados confortáveis ou confeccionados sob medida, coadjuvados com palmilhas. Neste estudo, $33 \%$ dos pacientes usavam calçado inapropriado durante a entrevista, número menor do que o encontrado em
Curitiba - PR em 2013, no qual 85\% dos pacientes faziam uso de calçado inadequado, ressaltando a importância das orientações em relação ao cuidado com os pés (CUBAS et al., 2013).

O trabalho da equipe multiprofissional, centrado no profissional da Enfermagem, é fundamental na potencialização do cuidado, através de orientações e educação em saúde, a fim de aumentar a aderência dos pacientes ao tratamento como um todo, e consequentemente atingir maior sucesso terapêutico.

\section{Conclusão}

Nesse estudo, o pé diabético foi mais prevalente em pacientes do sexo feminino, sendo a hipertensão arterial sistêmica e a neuropatia periférica os fatores de risco mais presentes. Além desses, foram observados outros fatores de risco modificáveis envolvidos no desenvolvimento do pé diabético, como tabagismo, sobrepeso/obesidade e sedentarismo.

Constatou-se que mesmo atendidos em centro com atendimento multidisciplinar, os pacientes apresentam mau controle glicêmico, o que corrobora a necessidade de aderência do paciente ao tratamento.

É fundamental ainda a instituição da educação em diabetes em todos os níveis de cuidado, para que a prevenção se dê de forma realmente eficaz. A orientação desses pacientes sobre cuidado com os pés é um importante instrumento na redução do risco de ulceração e amputação em membros inferiores.

\section{Diabetic Foot: where can we intervene?}

\section{Abstract}

This is a descriptive study, cross-cohort including 59 individuals with DM, who had diabetic foot as a complication treated at the Diabetic Foot Outpatient Clinic of Hiperdia center in the city of Juiz de Fora - MG, from July 2013 to July 2014. Results: The most prevalent predisposing conditions were hypertension (96.6\%), neuropathy (85.4\%) and dyslipidemia (69.5\%). It was also noted the presence of overweight / obesity (28.8\% / 50.8\%), smoking (42.4\%), physical inactivity (28.8\%) and poor glycemic control (HbA1c: $8.8 \% \pm 2.24$ ). Regarding foot care, $86.4 \%$ of participants said they were aware. Conclusion: It is fundamental the institution of diabetes education at all levels of care, so that prevention is to take really effective, since most risk factors are modifiable. The orientation of these patients about foot care is an important tool in reducing the risk of ulceration and amputation of lower limbs.

Key words: Diabetes Mellitus. Diabetic foot. Risk Factors. Self care. 


\section{REFÊRENCIS}

OLIVEIRA, J. E. P.; VENCIO, S. Diretrizes da Sociedade Brasileira de Diabetes: 2013-2014/Sociedade Brasileira de Diabetes ; São Paulo: AC Farmacêutica, 2014. Classificação etiológica do diabetes mellitus- p. 5-8.

PACE, A.E.; FOSS, M.C.; VIGO, K.O.; HAYASHIDA, M. Fatores de risco para complicações em extremidades inferiores de pessoas com Diabetes Mellitus. Rev. Bras. Enferm., v.55, n.5, p.514-521, 2002.

SANTOS, I. C. R. V. et al. Prevalência de pé diabético e fatores associados nas unidades de saúde da família da cidade do Recife, Pernambuco, Brasil, 2005. Cad. Saúde Pública vol.24 n.12, p.2861-2870, Rio de Janeiro, dez, 2008.

MICHELS, M.J. et al. Questionário de Atividades de Autocuidado com o Diabetes: tradução, adaptação e avaliaçãodas propriedades psicométricas. Arq Bras Endocrinol Metab. Vol. 54 n. 7. p.644-51, 2010.

GROSS, J.L.; NEHME, M. Detecção e tratamento das complicações crônicas do diabetes melito: Consenso da Sociedade Brasileira de Diabetes e Conselho Brasileiro de Oftalmologia. Ver. Ass Med Brasil, vol.45, n.3, p. 279-84, 1999.

\section{DEPARTAMENTO DE AÇÕES PROGRAMÁTICAS} ESTRTAÉGICAS,SECRETARIA DE POLÍTICAS DE SAÚDE. Ministério da Saúde. Plano de reorganização da atenção à hipertensão arterial e ao diabetes mellitus. Brasília: Ministério da Saúde; 2002.

CESCHINI, F.L.; ANDRADE, D.R.; OLIVEIRA, L.C.; ARAÚJO, J.J.F.; MATSUDO, V.K. Prevalence of physical inactivity and associated factors among high school students from state's public schools. J Pediatr (Rio J). vol.85 n. 4 p.301306, 2009 .

MENESES-GAYA, I.C. et al. As propriedades psicométricas do Teste de Fagerström para Dependência de Nicotina. J Bras Pneumol. vol.35 n. 1, p. 73-82, 2009.

FILHO, G.J. P. et al. Emprego do Questionário CAGE para detecção de transtornos de uso de álcool em pronto-socorro. Rev. Ass. Med. Brasil vol.45 n.1, p. 65-69, 2011.

BRASILEIRO, J.L. et al. Pé diabético: aspectos clínicos. Jornal vascular brasileiro vol.4 n.1, p.11-21, 2005.

NUNES, M.A.P. et al. Fatores predisponentes para amputação de membro inferior em pacientes diabéticos internados com pés ulcerados no estado de Sergipe. J Vasc Bras, vol.5 n.2, p. 123-30, 2006.

CUNHA-FILHO, I.T. et al. Correlação entre o índice tornozelo-braço antes e após teste de deslocamento bidirecional progressivo. J. vasc. bras., vol.6 n.4, p.332-38, 2007.
MAKDISSE, M. et al. Escore para rastrear idosos ( $>75$ anos) de alto risco para doença arterial periférica. Arq Bras Cardiol, vol.88 n.6, p.630-36, 2007.

ROCHA, R.M.; ZANETTT, M.L.; SANTOS, M.A.

Comportamento e conhecimento: fundamentos para prevenção do pé diabético. Acta Paul Enferm 2009; 22(1):17-23.

BORTOLETTO, M.S.S.; HADDAD, M.C.L.; KARINO, M.E. Pé diabético, uma avaliação sistematizada. Arq. Ciênc. Saúde Unipar, Umuarama, v. 13, n. 1, p. 37-43 jan./abr. 2009.

SBEM , Sociedade Brasileira de Endocrinologia e Metabologia. Obesidade, 2014. Disponível em http://www.endocrino.org.br/ obesidade/.

NORGREN, L.; HIATT, W.R.; DORMANDY J.A.; NEHLER M.R.; HARRIS K.A.; FOWKES, F.G.R. Inter-society consensus for the management of peripheral arterial disease (TASC II). J Vasc Bras.2007; 63 (Suppl 2):S194-s259.

MARTIN, I.S., BERALDO, A.A.; PASSERI, S.M.; FREITAS M.C.F.; PACE, A.E. Causas referidas para o desenvolvimento de úlceras em pés de pessoas com diabetes mellitus. Acta Paul Enferm. 2012;25(2):218-24.

PEDROSA, H.C.; ANDRADE, A. Consenso Internacional sobre Pé Diabético. Grupo de Trabalho Internacional sobre Pé Diabético. Versão Brasileira; 2001, SES-DF e Ministério da Saúde; versão 2003, 2007, 2009, 2011: disponível em www.idf. irg/bookshop.

ABBOT, C.A.; CARRINGTON, A.L.; ASHE, H. et al. The north-west diabetes foot care study: incidence of, and risk factors for, new diabetic foot ulceration in a community based patient cohort. Diabet Med. 2002; 20:377-84.

BOULTON, A.J.M. The diabetic foot: from art to science. The 18th Camillo Gol-gi Lecture. Diabetologia. 2004; 47: 1343-53.

CUBAS, M.R. et. al. Pé diabético: orientações e conhecimento sobre cuidados preventivos. Fisioter Mov. 2013 jul/set;26(3): 647-55.

INTERNACIONAL WORKING GROUP ON THE DIABETIC FOOT. Proceedings of the Internacional Working Group on the Diabetic Foot Meeting, Noordwijkerhout, the Netherlands, 1999-2003-2007-2011. 